\section{Author reply: Rituximab maintenance for follicular lymphoma}

\author{
Bruce D. Cheson
}

I appreciate the clarifications to my manuscript provided by Vidal and co-workers ${ }^{1}$ regarding their earlier publication. ${ }^{2}$ However, their analysis remains problematic. Most important is the preliminary nature of the data in several publications they included. Follicular lymphoma is an indolent disorder and prolonged follow-up is needed before broad conclusions about therapies can be drawn. This issue was no more evident than in the study of van Oers et al., where the earlier survival benefit disappeared with longer observation. ${ }^{3}$ Whereas they presented the data from the study by Hochster as two separate cohorts, with markedly different results, the data in their meta-analysis did not include the final publication. ${ }^{4}$ If they selectively choose to refer to individual cohorts in their analysis, why not separate out the R-CHOP from the CHOP arms of the data from van Oers. ${ }^{3}$ Doing so might help determine which populations of patients, if any, might benefit from maintenance rituximab. Even in the study by Ghielmini, ${ }^{5}$ at 8.9 years there was no significant survival benefit provided by maintenance for previously treated or untreated patients, only a trend towards an improved outcome.

There is an important difference between being statistically significant and being clinically meaningful. There is not a single study demonstrating a survival benefit from rituximab maintenance. The use of a metaanalysis of several negative studies to determine a positive result should not change patterns of care. It is critical to consider the best interests of the patient: maintenance therapy is expensive, inconvenient and, as the authors demonstrated in their study, associated with increased toxic effects. It may even compromise response to subsequent therapies. Thus, their recommendation for treatment of even relapsed and refractory follicular lymphoma patients with maintenance based on the currently available data cannot be accepted.

Georgetown University Hospital, Lombardi Comprehensive Cancer Center, 3800 Reservoir Road NW, Washington, DC 20007, USA. bdc4@georgetown.edu

doi:10.1038/nrclinonc.2009.161-c2

Competing interests

The author declares associations with Roche and Genentech.

1. Cheson, B. D. The case against rituximab maintenance. Nat. Rev. Clin. Oncol. 6, 622-624 (2009).

2. Vidal, L. et al. Rituximab maintenance for the treatment of patients with follicular lymphoma: systematic review and meta-analysis of randomized trials. J. Natl Cancer Inst. 101, 248-255 (2009).

3. Van Oers, M. H. J. et al. Rituximab maintenance treatment of relapsed/resistant follicular nonHodgkin's lymphoma: long-term outcome of the EORTC 20981 phase III randomized intergroup study [abstract 836]. Blood 112, 309-310 (2008).

4. Hochster, H. et al. Maintenance rituximab after cyclophosphamide, vincristine, and prednisone prolongs progession-free survival in advanced indolent lymphoma: results of the randomized phase III ECOG1496 study. J. Clin. Oncol. 27, 1607-1614 (2009).

5. Ghielmini, M. E. et al. Long-term follow-up of patients with follicular lymphoma (FL) receiving single agent rituximab at two different schedules in study SAKK 35/98 [abstract 8512]. J. Clin. Oncol. 27 (Part I), 437s (2009). 